\title{
Módulo de elasticidade de grãos de milho submetidos a impactos mecânicos
}

\author{
Solenir Ruffato ${ }^{1}$, Sandra M. Couto² \& Daniel M. de Queiroz ${ }^{3}$ \\ 1 DEA/UFV. CEP 365701-000, Viçosa, MG. Fone: (31) 3899-2729. E-mail: sruffato@alunos.ufv.br (Foto) \\ 2 DEA/UFV. E-mail: scouto@mail.ufv.br \\ 3 DEA/UFV. E-mail: queiroz@mail.ufv.br
}

Protocolo $089-13 / 07 / 2000$

\begin{abstract}
Resumo: Neste trabalho investigou-se a viabilidade de se obter o módulo de compressão de grãos de milho, utilizando-se dados experimentais de força versus tempo, provenientes de testes de impacto, juntamente com uma análise estrutural elástica do processo. Os módulos de elasticidade foram determinados para grãos, a diferentes teores de umidade, submetidos a impactos de diferentes velocidades, e obtidos por um processo de otimização por meio da técnica de elementos finitos. Dois tipos de módulo foram avaliados: (a) um módulo efetivo para todo o grão e (b) um módulo para cada uma das três regiões, com diferentes características, segundo as quais o grão foi dividido. $O$ teor de umidade e a velocidade de impacto influenciaram nos valores dos módulos. Módulos para grãos a $13,4 \%$ base úmida (b.u.) foram maiores do que para aqueles a $20,0 \%$ b.u. A análise realizada (elástica) mostrou-se ser mais adequada na obtenção de módulos de elasticidade de grãos a 13,4\% b.u.; neste teor, os grãos apresentam características elásticas mais pronunciadas que quando a $20,0 \%$ b.u. e, nos grãos com altos teores de umidade, as características viscoelásticas tornam-se predominantes.
\end{abstract}

Palavras-chave: módulo de elasticidade, otimização, elementos finitos

\section{Modulus of elasticity of shelled corn submitted to mechanical impacts}

\begin{abstract}
In this study the viability of obtaining the corn compression modulus through an elastic structural analysis was investigated using force versus time data from grain impact tests. The moduli of elasticity of shelled corn at different moisture contents submitted to various impact velocities were determined. The moduli were obtained through an optimization process using the finite element technique. Two kinds of modulus were obtained: (a) an effective modulus for the grain and (b) a modulus for each one of the three regions, with different characteristics, in which the grain was divided. The moisture content and the impact velocity affected the modulus values. The moduli values for grains at $13.4 \%$ wet basis (w.b.) were higher than those for grains at $20.0 \%$ w.b. The analysis used for the modulus seems to be more adequate for grains at $13.4 \%$ w.b. The grains at this moisture content present elastic characteristics while those at $20.0 \%$ w.b. present a viscoelastic behavior.
\end{abstract}

Key words: elastic modulus, optimization, finite elements

\section{INTRODUÇ̃̃O}

Durante o processamento e as operações de manuseio, grãos de milho são submetidos a forças de compressão dinâmicas devido, geralmente, a impactos que resultam em distribuições dinâmicas de tensões e de deformações no seu interior.

Segundo Goldsmith (1960) quando um corpo (não rígido) é impactado, a perturbação gerada no ponto de contato propaga-se no interior do material com velocidade finita e sua reflexão nas superfícies que contornam o material produz oscilações ou vibrações no sólido e as diversas seções do corpo não ficam simultaneamente expostas à mesma ação da força. A propagação dessas ondas depende do tipo de material do qual o corpo é constituído.
Segundo Ferry (1970) a teoria clássica da elasticidade lida com propriedades mecânicas de sólidos, para os quais a tensão é sempre diretamente proporcional à deformação (lei de Hooke). A teoria clássica da hidrodinâmica trabalha com propriedades de líquidos viscosos, para os quais a tensão é sempre diretamente proporcional à taxa de deformação, mas independente da deformação propriamente dita (lei de Newton). Embora essas categorias sejam idealizações, existem sólidos que, para deformações infinitesimais, comportam-se, aproximadamente, como elástico, e semelhante a muitos fluidos que se aproximam da lei de Newton, quando submetidos a taxas de deformação infinitesimais. Quando a relação entre a tensão e a deformação de um corpo é dependente do tempo, o material é dito viscoelástico; combina características de sólido e líquido. 
Zoerb \& Hall (1960) investigaram algumas propriedades mecânicas de espécimes de grãos de milho sob compressão, entre placas paralelas, a velocidades que variaram de 0,198 a $1,186 \mathrm{~cm} \mathrm{~min}^{-1}$, e observaram comportamento elástico desses materiais na primeira porção de deformação, exceto quando o produto se encontrava a altos teores de umidade, caso em que os grãos se tornam menos elásticos e mais viscosos. A taxa de compressão não afetou a resistência compressiva dos grãos a teores de umidade baixos e médios, mas diferenças significativas do efeito da velocidade foram detectadas para grãos a altos teores de umidade. Com base nas observações de um grande número de pesquisadores, o comportamento mecânico de grãos agrícolas é considerado como o de materiais viscoelásticos.

As propriedades mecânicas que governam a forma pela qual um material transmite vibrações são, basicamente, sua massa específica, elasticidade e friç̧ão interna (Mohsenin, 1986). Quando um corpo viscoelástico é submetido a uma tensão que oscila senoidalmente, a deformação resultante não estará em fase com a tensão, como seria no caso de um corpo elástico, mas parte da energia introduzida no sistema é armazenada e recuperada em cada ciclo e a outra parte é dissipada em forma de calor. Na determinação do "módulo de compressão" de um material elástico, a taxa com a qual a deformação é aplicada ao material é irrelevante, ou seja, qualquer método de aplicação de forças, estático ou dinâmico, conduz a resultados similares.

A modelagem da ação de carregamentos dinâmicos em grãos agrícolas requer o conhecimento de suas propriedades viscoelásticas. Dentre os métodos experimentais para caracterização viscoelástica de materiais, cita-se o do módulo dinâmico, que consiste na determinação do módulo de elasticidade em função da freqüência da deformação senoidal.

As pesquisas com grãos têm mostrado que, à medida em que se aumenta a freqüência da excitação imposta ao produto, $o$ grão responde muito mais elasticamente, ou seja, existe um amortecimento menor quando este é submetido a freqüências mais altas. Tem sido mostrado, também, que os grãos são mais elásticos quando se encontram a teores de umidade mais baixos e, quando estão com umidades mais altas, a dissipação de energia é maior. Resumindo, os grãos respondem mais elasticamente às forças de impacto quando se encontram a baixas umidades e são submetidos a altas freqüências.

Muitas pesquisas foram realizadas visando-se à determinação de módulos de elasticidade de grãos sob compressões quase estáticas. Zoerb \& Hall (1960) usando uma taxa de aplicação de força de $0,678 \mathrm{~cm} \mathrm{~min}^{-1}$, determinaram os módulos de elasticidade de espécimes de grãos de milho numa faixa de umidade de 13,3 a 18,7\% b.u. Moreira (1980) determinou o módulo de elasticidade de diferentes regiões de um grão de milho. Os testes foram realizados com o produto a teores de umidade variando de 13,4 a $29,3 \%$ b.u. comprimidos a uma taxa de $0,051 \mathrm{~cm} \mathrm{~min}^{-1}$.

Poucas informações existem sobre módulos de elasticidade dinâmicos de grãos agrícolas. Wen \& Mohsenin (1970) determinaram os módulos de elasticidade dinâmicos, obtidos por um processo de aplicação senoidal de forças, para espécimes de endosperma de grãos de milho.

Resultados experimentais têm mostrado haver certa proporcionalidade entre os valores dinâmicos e estáticos do módulo de compressão. Bartsch (1979) trabalhando com soja a diferentes teores de umidade, verificou que os valores dos módulos de elasticidade dinâmicos, obtidos por meio de testes ultra-sônicos, usando uma freqüência de excitação de $30 \mathrm{MHz}$ eram, aproximadamente, 10 vezes maiores do que aqueles determinados por processos de compressão quase estáticos. Os módulos dinâmicos decresceram, ligeiramente, com um aumento no teor de umidade do produto. Os seus valores dinâmicos foram similares aos determinados por Hooki \& Monita (1975) e Hooki (1979) para o mesmo produto, usando-se uma freqüência de $200 \mathrm{kHz}$. Chen et al. (1996) trabalhando com melão a uma freqüência de excitação de $800 \mathrm{~Hz}$, observaram que o módulo de elasticidade dinâmico era seis vezes maior do que o estático. Por outro lado, Chen et al. (1992) verificaram que o módulo de elasticidade dinâmico de maçã era 3,7 vezes maior que o estático.

Embora haja diferenças entre os grãos de milho usados nos testes, os módulos estáticos encontrados por Moreira (1980) são inferiores àqueles determinados por Zoerb \& Hall (1960). Em termos de módulo dinâmico, nota-se que as relações entre os módulos dinâmicos e os estáticos, obtidos por Zoerb \& Hall (1960) são iguais a 1,$43 ; 0,80 ; 0,85$ e 0,98 , para teores de umidade próximos de 13,$3 ; 14,5 ; 15,8$ e $18,7 \%$ b.u., respectivamente.

As técnicas usadas para a determinação do módulo de compressão de grãos sob a ação de carregamentos dinâmicos são, em geral, sofisticadas, e um dos fatores limitantes é o pequeno tamanho dos grãos que, na maioria das vezes, danifica os sensores utilizados. A investigação de técnicas mais simples para a determinação de módulos de compressão seria de grande importância nas análises para predições de fraturas em um produto sob a ação de forças dinâmicas. Tal técnica poderia basear-se em dados experimentais mais fáceis de serem obtidos como, por exemplo, variações temporais das forças que atuam no produto durante um impacto.

Com base nessas idéias, este trabalho teve por objetivo investigar a viabilidade de se obter o módulo de compressão de um grão, utilizando-se dados experimentais de força versus tempo, provenientes de testes de impacto, juntamente com uma análise estrutural elástica do processo.

\section{MATERIAL E MÉTODOS}

Os dados experimentais utilizados neste trabalho foram extraídos de Moreira (1980) e se referem a grãos de milho a teores de umidade de 13,4 e 20,0\% b.u., impactados, segundo o esquema mostrado na Figura 1 por uma superfície rígida, a velocidades de 10, 14 e $18 \mathrm{~m} \mathrm{~s}^{-1}$.

Por meio de um processo de otimização e usando-se a técnica de elementos finitos para modelar o impacto, procurou-se ajustar os valores de módulos de elasticidade do grão de tal maneira, que os valores simulados das forças na região de contato estivessem o mais próximo possível daqueles de força máxima determinados experimentalmente.

Foram realizadas oito otimizações, sendo que, para cada um dos seis experimentos combinando "teor de umidade do grão e velocidade de impacto", dois tipos de otimização foram realizados: $\left(T_{1}\right)$ considerando-se um único módulo de elasticidade para cada grão e $\left(T_{2}\right)$ e três módulos, um para cada região à qual se havia determinado o módulo de elasticidade estático (Figura 1). 


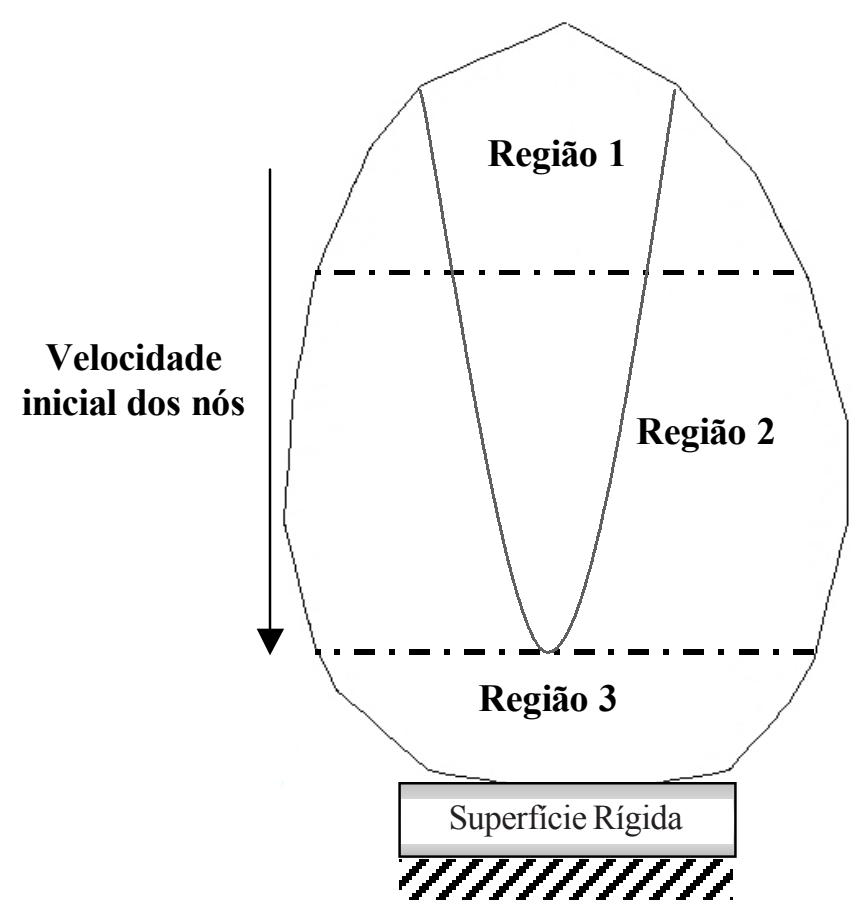

Figura 1. Regiões do grão de milho usadas na determinação de módulos de elasticidade

O procedimento usado na otimização dos módulos de elasticidade do grão de milho encontra-se esquematizado na Figura 2 e consiste, basicamente, de um programa principal, ao qual se introduziu uma sub-rotina desenvolvida para viabilizar o processo de otimização. O programa principal usado, similar ao exposto por Ruffato et al. (1999) proporciona, em intervalos de tempo pré-definidos, os valores de deslocamento, deformações e tensões resultantes em um grão de milho impactado por uma placa rígida (Figura 1). Usando-se esses resultados, determina-se a força resultante nos nós do grão em contato direto com a placa, força de reação $\mathrm{F}_{\mathrm{D}}$.

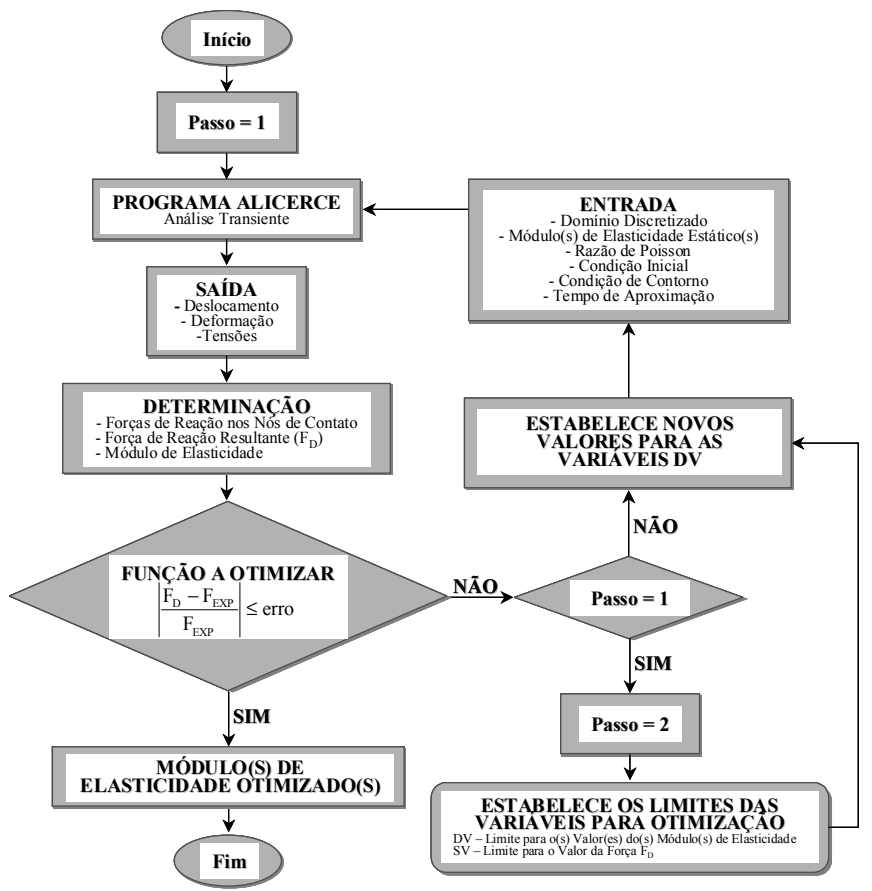

Figura 2. Esquema do procedimento usado na otimização do(s) módulo(s) de elasticidade
Para a execução do programa principal são necessários os seguintes parâmetros de entrada: propriedades do material (módulo de elasticidade, massa específica e razão de Poisson), condição inicial (velocidade em todos os nós, considerada como a de impacto), condições de contorno (deslocamento, considerado nulo nos nós do grão em contato com a superfície rígida) e o tempo de aproximação (tempo em que a força resultante nos nós do grão, em contato com a superfície, atinge um valor máximo durante o impacto).

No processo de otimização e usando-se o Método de Aproximação do Subproblema (Subproblem Approximation Method) definiu-se, inicialmente, uma função para ser minimizada $\left(\mathrm{E}_{\mathrm{r}}\right)$ :

$$
\mathrm{E}_{\mathrm{r}}=\left|\frac{\mathrm{F}_{\mathrm{D}}-\mathrm{F}_{\mathrm{EXP}}}{\mathrm{F}_{\mathrm{EXP}}}\right|
$$

Esta função corresponde ao erro relativo entre os valores das forças de reação, $\mathrm{F}_{\mathrm{D}}$, calculados pelo programa principal, e o determinado experimentalmente $\left(\mathrm{F}_{\mathrm{EXP}}\right)$ tomando-se como referência o valor experimental. Considerou-se que a quantidade $\mathrm{E}_{\mathrm{r}}$ é uma função da força de reação, $\mathrm{F}_{\mathrm{D}}$, que, por sua vez, é uma função dos valores dos módulos de elasticidade. Desta forma, no processo de otimização três tipos de variáveis foram usados: (a) $E_{r}$, função objetivo, que é a grandeza que se deseja otimizar; (b) DV, quantidades independentes, denominadas variáveis de projeto, cujos valores são modificados até atingir-se o valor ótimo da função objetivo, em que o domínio de variação das DV, durante o processo de otimização, ficou restrito pela especificação de seus valores limites (mínimo e máximo); e (c) $\mathrm{SV}$, variável dependente, denominada variável de estado, calculada em função das DV, obtidas a partir do programa principal. Valores mínimo e máximo para a variável força de reação $\left(\mathrm{F}_{\mathrm{D}}\right)$, também foram especificados para o direcionamento do processo de otimização.

Para dada situação de impacto, combinando-se "teor de umidade do grão e velocidade de impacto", o programa principal era executado usando-se, inicialmente, um valor de módulo de elasticidade definido como o produto entre uma variável "Fator" e o módulo estático. "Fator" foi assim considerada a variável DV no processo de otimização, a qual recebia um valor inicial igual a um. $\mathrm{O}$ valor da força de reação, $\mathrm{F}_{\mathrm{D}}$, em um tempo igual ao de aproximação (experimental) era, então, determinado e usado na estimativa de $\mathrm{E}_{\mathrm{r}}$. Se o valor desta grandeza fosse menor ou igual a 0,1 (incerteza envolvida na instrumentação experimental para determinação das forças) o processo de otimização era concluído; caso contrário, novos valores de "Fator" eram introduzidos e o programa principal executado até que a condição restritiva para $\mathrm{F}_{\text {Ótimo }}$ fosse satisfeita.

Nas otimizações, a faixa de variação permitida aos valores de "Fator" (implicando nas variações dos módulos de elasticidade) foi de 0,1 a 10 . No caso da otimização do tipo $T_{1}$, a variável "Fator" foi multiplicada pelo menor valor dos módulos estáticos das três regiões do produto e na otimização do tipo $\mathrm{T}_{2}$, o módulo estático de cada uma das três regiões do grão foi multiplicado pelo mesmo valor de "Fator". Em todos os casos, tomou-se como os limites inferior e superior para a força de reação, $\mathrm{F}_{\mathrm{D}}$, o valor experimental \pm 1 , respectivamente. 


\section{RESULTADOS E DISCUSSÃO}

A Tabela 1 apresenta os valores dos parâmetros utilizados como entrada para execução do programa principal e na realização do processo de otimização.

Na Figura 3 são apresentados os módulos de elasticidade de grãos de milho, a diferentes teores de umidade e impactados a três velocidades, usando-se os procedimentos (a) compressão estática, (b) otimização de um único módulo para todo o grão $\left(\mathrm{T}_{1}\right)$ e (c) otimização de um módulo para cada região do grão $\left(\mathrm{T}_{2}\right)$.

Na obtenção de todos os módulos foram usados os tempos de aproximação médios experimentais, exceto no caso das otimizações para grãos a $20,0 \%$ b.u. impactados a $14 \mathrm{~m} \mathrm{~s}^{-1}$. Neste caso, quando então se usou o tempo médio experimental na simulação, o valor máximo simulado atingido pela força de reação foi de $154,4 \mathrm{~N}$ e as variações realizadas nos valores dos três módulos não a elevaram para próximo de 171,4 N (máxima experimental) como esperado. A resposta do sistema à utilização de outros tempos de aproximação, percentuais abaixo e acima do tempo de aproximação médio, foi investigada e é apresentada na Figura 4.

Ao se usar, nas simulações, valores de 1 a $4 \%$ inferiores aos tempos experimentais, obtiveram-se valores muito altos para a força de reação; para um valor de $5 \%$ abaixo do tempo experimental é que ocorreu a força máxima esperada. Observa-se,

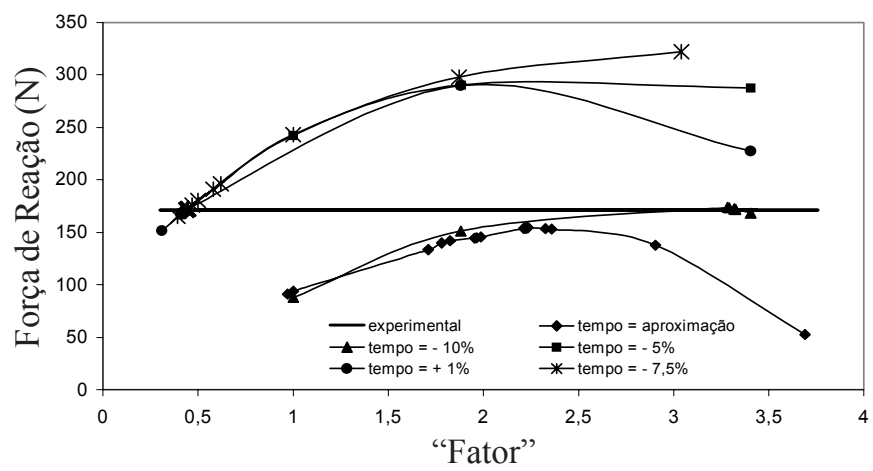

Figura 4. Variação da força de reação com mudanças nos valores de "Fator" para impactos de grão de milho a 20,0 \% b.u. a uma velocidade de $14 \mathrm{~m} \mathrm{~s}^{-1}$

na Figura 4, que os valores resultantes da variável "Fator", fator multiplicativo para os módulos de elasticidade, foram muito próximos e iguais a 0,$43929 ; 0,43928$ e 0,43624 , ao serem usados tempos de 5 e 7,5\%, abaixo do valor experimental, e de $1 \%$, acima do experimental, respectivamente. Ressalta-se que, apesar da curva para o tempo de $1 \%$ acima do experimental indicar tendência de tocar, em um segundo ponto, a reta correspondente à força de 171,4 N (experimental) isto não acontece; quando "Fator" excede a 3,5 , os valores da força de reação são altíssimos e, ao se usar um valor de tempo $10 \%$ abaixo do experimental, houve aumento razoável no valor da variável multiplicativa, que passou

Tabela 1. Valores dos parâmetros usados no processo de otimização

\begin{tabular}{|c|c|c|c|c|c|c|c|}
\hline \multirow{3}{*}{$\begin{array}{l}\text { Região } \\
\text { do Grão }\end{array}$} & Módulo & tático (MPa) & \multirow{3}{*}{$\begin{array}{l}\text { Velocidade } \\
\left(\mathrm{m} \mathrm{s}^{-1}\right)\end{array}$} & \multirow{2}{*}{\multicolumn{4}{|c|}{ Teor de Umidade (\% b.u.) }} \\
\hline & \multicolumn{2}{|c|}{ Teor de Umidade (\% b.u.) } & & & & & \\
\hline & 13,4 & 20,0 & & 13,4 & 20,0 & 13,4 & 20,0 \\
\hline 1 & $92,3(46,6)^{*}$ & $61,4(44,0)$ & 10 & $157,3(4,5)$ & $101,2(5,9)$ & $26,3(15,2)$ & $37,3(21,4)$ \\
\hline 2 & $290,1(57,6)$ & $135,7(42,0)$ & 14 & $199,3(4,5)$ & $171,4(4,1)$ & $28,2(28,4)$ & $42,2(16,6)$ \\
\hline 3 & $119,7(50,1)$ & $91,3(62,4)$ & 18 & $252,0(3,6)$ & $194,0(3,6)$ & $26,2(15,3)$ & $39,9(17,5)$ \\
\hline
\end{tabular}

* Valor entre parênteses representa o coeficiente de variação (\%)



Figura 3. Valores dos módulos de elasticidade (M.E.) determinados por compressão estática e pelos processos de otimização: $\left(T_{1}\right)$ um módulo equivalente para todo o grão, e $\left(\mathrm{T}_{2}\right)$ um módulo para cada uma das três regiões do grão 
a ser igual a 3,3262 e, conseqüentemente, isto alteraria em muito os valores dos módulos elásticos das regiões do grão. Adotou-se como tempo de aproximação nas simulações, para grãos a $20 \%$ b.u. impactados a $14 \mathrm{~m} \mathrm{~s}^{-1}$, um tempo igual a $1 \%$ acima do médio experimental, por ser o mais próximo daquele obtido por Moreira (1980) e por mostrar coerência com os valores de "Fator" obtidos para os tempos de 5 e 7,5\% abaixo do experimental.

O comportamento dos módulos otimizados, Figura 3, parece diferir do teor de umidade do grão, da velocidade de impacto e do processo usado na otimização. Quando são considerados os dois processos de otimização, nota-se que, para todas as velocidades de impacto, os módulos determinados para grãos a $13,4 \%$ b.u. de umidade são maiores do que para aqueles a $20,0 \%$ b.u., enquanto no caso de grãos a $13,4 \%$ b.u., o menor módulo determinado pelo processo $T_{1}$ foi para impactos a uma velocidade de $18 \mathrm{~m} \mathrm{~s}^{-1}$, enquanto pelo processo $T_{2}$ impactos nesta velocidade resultaram nos maiores módulos para todas as regiões do grão.

Quando os grãos se encontram a 20,0\% b.u., os valores dos módulos, provenientes dos dois processos de otimização, parecem não ser afetados pela intensidade de impacto. Observa-se que os valores dos módulos de elasticidade resultantes são menores que aqueles determinados experimentalmente pelo método estático.

A Tabela 2 apresenta os valores das forças de reação resultantes nos dois processos de otimização, os erros envolvidos e a variável "Fator", que corresponde à relação entre o módulo simulado e o estático. No caso da otimização de um único módulo para o grão, foi utilizado, para cálculo de "Fator", o menor módulo de elasticidade do produto e, na otimização para três módulos, o módulo de elasticidade estático da região correspondente (Tabela 1). Por outro lado, os módulos dinâmicos determinados por Wen \& Mohsenin (1970) foram 1,60 e 7,61 vezes maiores do que os módulos equivalentes (um único módulo para todo o grão) obtidos neste trabalho para grãos a 13,4 e 20,0\% b.u., respectivamente, considerando-se uma média para todas as velocidades de impacto. Ressalta-se que os valores obtidos por esses autores são para espécimes do endosperma duro do grão de milho. Se forem considerados os módulos determinados para a região 2 , onde se concentra a maior parte do endosperma duro, ter-se-á que os módulos obtidos por eles são 0,93 e 4,99 vezes aqueles obtidos neste trabalho para grãos com umidade de 13,4 e 20,0\% b.u., respectivamente.

Considerando-se que: (a) os valores dinâmicos usados nas comparações foram obtidos em diferentes freqüências de

Tabela 2. Forças de reação obtidas pelos dois processos de otimização e fatores multiplicativos dos módulos simulados

\begin{tabular}{|c|c|c|c|c|c|c|c|}
\hline \multirow{2}{*}{$\begin{array}{l}\text { Umidade } \\
\text { (\% b.u.) }\end{array}$} & \multirow{2}{*}{$\begin{array}{l}\text { Velocidade } \\
\qquad\left(\mathrm{m} \mathrm{s}^{-1}\right)\end{array}$} & \multicolumn{2}{|c|}{$\begin{array}{c}\text { Força } \\
\text { Simulada }(\mathrm{N})\end{array}$} & \multicolumn{2}{|c|}{$\begin{array}{c}\text { Erro } \\
\text { Relativo (\%) }\end{array}$} & \multicolumn{2}{|c|}{ "Fator" } \\
\hline & & (T1) & (T2) & (T1) & $(\mathrm{T} 2)$ & (T1) & (T2) \\
\hline \multirow{3}{*}{13,4} & 10 & 157,42 & 157,37 & 0,076 & 0,045 & 4,45 & 1,80 \\
\hline & 14 & 199,31 & 199,30 & 0,005 & 0,000 & 3,82 & 1,54 \\
\hline & 18 & 251,98 & 252,16 & 0,008 & 0,063 & 3,57 & 5,08 \\
\hline \multirow{3}{*}{20,0} & 10 & 101 & 10 & 0,089 & 0,069 & 0,35 & 0,25 \\
\hline & 14 & 171,37 & 171,50 & 0,018 & 0,058 & 0,69 & 0,43 \\
\hline & 18 & 194,16 & 193,97 & 0,082 & 0,015 & 0,45 & 0,31 \\
\hline
\end{tabular}

excitação das simuladas pelos impactos neste trabalho; (b) a região 2 contém outros materiais além do endosperma duro e (c) a geometria do grão simulado pode não ser aquela correspondente aos dados de força e tempo de aproximação usados, a obtenção do módulo elástico, usando-se elementos finitos, parece ser eficaz para grãos a baixos teores de umidade. Nestas condições, o grão, por apresentar características mais elásticas, adapta-se melhor ao tipo de análise realizada (elástica) para a obtenção dos módulos.

\section{CONCLUSÕES}

Combinando-se teor de umidade do grão e velocidade de impacto, dois processos de otimização foram realizados:

1. Um único módulo de elasticidade para cada grão.

2. Três módulos, considerando-se regiões do grão com características diferenciadas, em que:

- os valores dos módulos de elasticidade otimizados diferiram com o teor de umidade, a velocidade de impacto e o processo usado na otimização;

- para todas as velocidades de impacto, os módulos de elasticidade foram superiores para grãos com menor teor de umidade ( $13,4 \%$ b.u.) nos dois processos de otimização;

- a força de impacto não demonstrou ter efeito significativo sobre os valores dos módulos, quando os grãos se encontravam a $20,0 \%$ b.u.;

- a técnica de elementos finitos na obtenção do módulo elástico mostrou ser eficaz para grãos a baixos teores de umidade, considerando-se: diferentes freqüências de excitação das simuladas pelos impactos, comparadas aos valores dinâmicos; limite das regiões do grão não coincidentes com a de um grão real; e geometria do grão simulado não correspondente ao experimental.

\section{LITERATURA CITADA}

Bartsch, J.A. Internal damage and dynamic stress levels resulting from impact loading of soybean kernels. West Lafayette, EUA: Purdue University, 1979. 129p. Ph.D. Thesis.

Chen, H.; Baerdemaeker, J. de; Bellon, V. Finite element study of the melon for nondestructive sensing of firmness. Transactions of the ASAE, St. Joseph, v.39, n.3, p.1057-1065, 1996.

Chen, P.; Sun, Z.; Huarng, L. Factors affecting acoustic responses of apples. Transactions of the ASAE, St. Joseph, v.35, n.6, p.1915-1920, 1992.

Ferry, J.D. Viscoelastic properties of polymers. 2 ed. New York: John Wiley \& Sons, Inc. 1970. 351p.

Goldsmith, W. Impact: The theory and physical behavior of colliding solids. London: Edward Arnold Publishers, Ltd., 1960.260p.

Hooki, M. Physical properties of soybeans. Seminar Presented at Purdue University. Department of Agricultural Engineering. April 3, 1979.

Hooki, M.; Monita, K. Mechanical properties of soybeans by means of ultrasonic measurements. The Bulletin of the Faculty of Agriculture, Michigan University. n.49, p.295-300, 1975.

Mohsenin, N.N. Physical properties of plant and animal materials. 2. ed. New York: Gordon and Breach Science Publishers, Inc. 1986.742p. 
Moreira, S.M.C. Impact properties and impact damage to corn. West Lafayette, EUA: Purdue University, 1980. 108p. Ph.D. Thesis.

Ruffato, S.; Couto, S.M.; Queiroz, D.M. de. Resposta de grão de milho a impactos - análise por elementos finitos. In: Congresso Brasileiro de Engenharia Agrícola, 28, 1999, Pelotas. Anais... Pelotas: Universidade Federal de Pelotas. CD-Rom
Wen, P.R.; Mohsenin, N.N. Applications of pulse techniques for determination of the elastic modulus of yellow corn. Material Research and Standards, v.10, n.12, p.25-27, 1970.

Zoerb, G.C.; Hall, C.W. Some mechanical and rheological properties of grains. Journal of Agricultural Engineering Research, v.5, n.1, p.83-93, 1960. 